\title{
Clinical and immunological responses after CD30-specific chimeric antigen receptor-redirected lymphocytes
}

\author{
Carlos A. Ramos, ${ }^{1,2}$ Brandon Ballard, ${ }^{1}$ Huimin Zhang, ${ }^{1}$ Olga Dakhova, ${ }^{1}$ Adrian P. Gee, ${ }^{1}$ Zhuyong Mei, ${ }^{1}$ Mrinalini Bilgi, ${ }^{1}$ \\ Meng-Fen Wu, ${ }^{3}$ Hao Liu, ${ }^{2,3}$ Bambi Grilley, ${ }^{1,4}$ Catherine M. Bollard, ${ }^{1,2,4}$ Bill H. Chang, ${ }^{5}$ Cliona M. Rooney, ${ }^{1,6,7}$ Malcolm K. Brenner, ${ }^{1,2,4}$ \\ Helen E. Heslop, ${ }^{1,2,4}$ Gianpietro Dotti, ${ }^{1,2}$ and Barbara Savoldo ${ }^{1,4}$ \\ ${ }^{1}$ Center for Cell and Gene Therapy, Baylor College of Medicine, Houston Methodist Hospital and Texas Children's Hospital, Houston, Texas, USA. ${ }^{2}$ Department of Medicine, ${ }^{3}$ Biostatistics Shared Resource, Dan \\ L. Duncan Cancer Center, and ${ }^{4}$ Department of Pediatrics, Baylor College of Medicine, Houston, Texas, USA. ${ }^{5}$ Division of Pediatric Hematology and Oncology, Oregon Health and Science University, Portland, \\ Oregon, USA. ${ }^{6}$ Department of Pathology and Immunology and 'Department of Molecular Virology and Microbiology, Baylor College of Medicine, Houston, Texas, USA.
}

\begin{abstract}
BACKGROUND. Targeting CD30 with monoclonal antibodies in Hodgkin lymphoma (HL) and anaplastic large cell lymphoma $(A L C L)$ has had profound clinical success. However, adverse events, mainly mediated by the toxin component of the conjugated antibodies, cause treatment discontinuation in many patients. Targeting CD30 with T cells expressing a CD30specific chimeric antigen receptor (CAR) may reduce the side effects and augment antitumor activity.
\end{abstract}

\begin{abstract}
METHODS. We conducted a phase I dose escalation study in which 9 patients with relapsed/refractory HL or ALCL were infused with autologous T cells that were gene-modified with a retroviral vector to express the CD30-specific CAR (CD30. CAR-Ts) encoding the CD28 costimulatory endodomain. Three dose levels, from $0.2 \times 10^{8}$ to $2 \times 10^{8} \mathrm{CD} 30 . \mathrm{CAR}-\mathrm{Ts} / \mathrm{m}^{2}$, were infused without a conditioning regimen. All other therapy for malignancy was discontinued at least 4 weeks before CD30. CAR-T infusion. Seven patients had previously experienced disease progression while being treated with brentuximab.
\end{abstract}

\begin{abstract}
RESULTS. No toxicities attributable to CD30.CAR-Ts were observed. Of 7 patients with relapsed HL, 1 entered complete response (CR) lasting more than 2.5 years after the second infusion of CD30.CAR-Ts, 1 remained in continued CR for almost 2 years, and 3 had transient stable disease. Of 2 patients with ALCL, 1 had a CR that persisted 9 months after the fourth infusion of CD30.CAR-Ts. CD30.CAR-T expansion in peripheral blood peaked 1 week after infusion, and CD30.CAR-Ts remained detectable for over 6 weeks. Although CD30 may also be expressed by normal activated T cells, no patients developed impaired virus-specific immunity.
\end{abstract}

CONCLUSION. CD30.CAR-Ts are safe and can lead to clinical responses in patients with HL and ALCL, indicating that further assessment of this therapy is warranted.

TRIAL REGISTRATION. ClinicalTrials.gov NCT01316146.

FUNDING. National Cancer Institute (3P50CA126752, R01CA131027 and P30CA125123), National Heart, Lung, and Blood Institute (R01HL114564), and Leukemia and Lymphoma Society (LLSTR 6227-08).

\section{Introduction}

Provision of antigen specificity to T cells by insertion of chimeric antigen receptor (CAR) molecules offers an effective therapeutic option for patients with $\mathrm{B}$ cell lymphoid malignancies (1-10). The selective expression of the CD19 antigen by normal and malignant $\mathrm{B}$ cells has fueled the development of CAR-T therapy, since the associated $B$ cell aplasia is a manageable side effect if objective and sustained clinical responses are achieved (11-13). Similarly, the limited expression of CD30 by normal tis-

Conflict of interest: While this clinical trial was being conducted, the Center for Cell and Cene Therapy at Baylor College of Medicine had a Collaborative Research Agreement with Celgene Corp. and Bluebird Bio.

Submitted: March 29, 2017; Accepted: June 29, 2017.

Reference information: / Clin Invest. 2017;127(9):3462-3471.

https://doi.org/10.1172/JCI94306. sues and its consistent overexpression by Hodgkin lymphoma (HL) and anaplastic large cell lymphoma (ALCL) tumor cells (14) have encouraged the development of CD30-directed CAR-T cell (CD30.CAR-T) therapy. In addition, the observed lack of "on-target" toxicities with toxin-conjugated CD30-specific monoclonal antibodies (15), such as brentuximab, together with the promising outcomes produced by this drug (16-18), further boosted the interest in developing CD30.CAR-Ts to improve the rate of clinical responses.

The proof of concept that HL is susceptible to immunemediated killing originates from the remarkable responses observed in patients receiving ex vivo expanded T cells targeting virus-associated antigens, such as Epstein-Barr virus (EBV) in EBV-associated HL and non-Hodgkin lymphoma (19-21). Targeting CD30 with CD30.CAR-Ts offers the opportunity to rapidly generate tumor-specific $\mathrm{T}$ cells in all patients with $\mathrm{HL}$ 


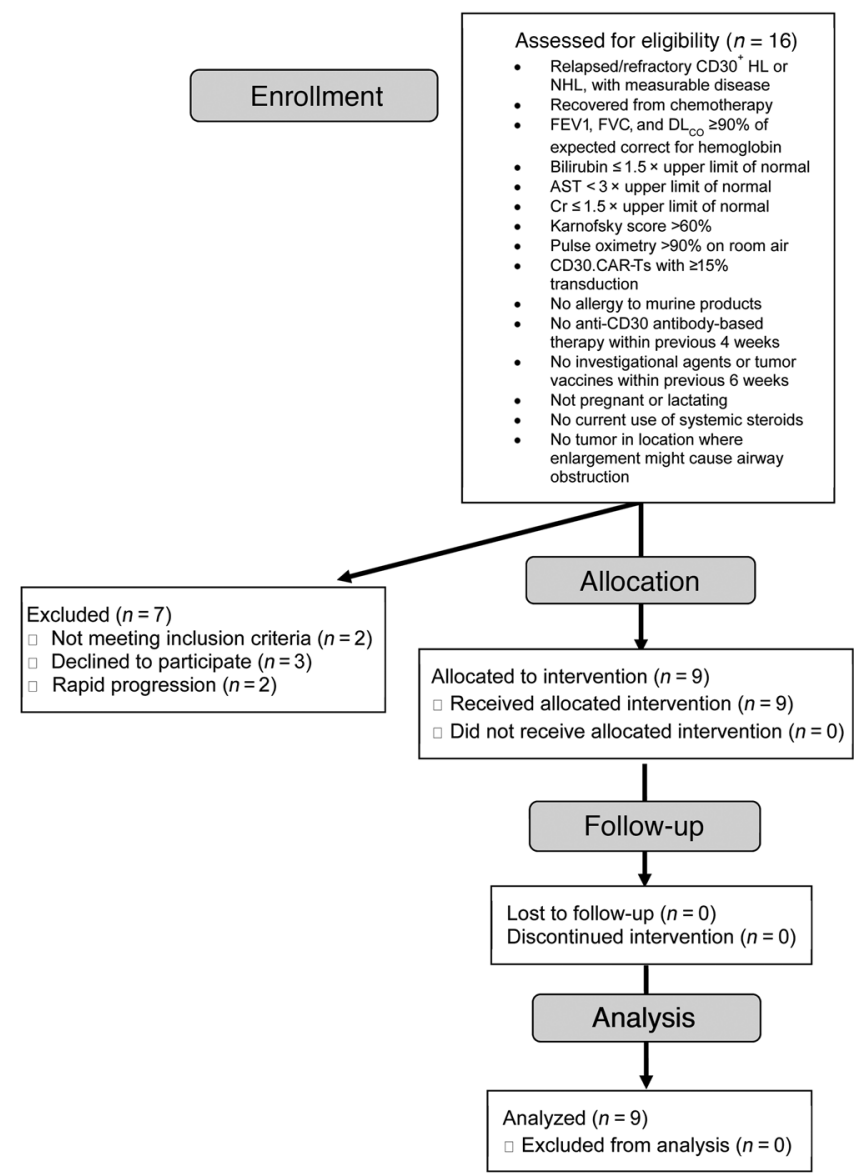

Figure 1. Flowchart of clinical trial NCT01316146 (CART CD30). FEV1, forced expiratory volume in 1 second; FVC, forced vital capacity; DLCO, diffusing capacity of the lungs for carbon monoxide; AST, aspartate aminotransferase; $\mathrm{Cr}$, creatinine.

and ALCL, independent of their association with EBV. In addition, tumor recognition by CD30.CAR-Ts is MHC-unrestricted and thus overcomes potential tumor escape secondary to HLA downregulation or defective antigen processing (1). Finally, CAR engineering couples antigen recognition with $\mathrm{T}$ cell costimulation, providing a major advantage compared with canonical TCR recognition, as tumor cells may lack the expression of costimulatory molecules. The recent success of PD1 blockade in HL (22-24) has further emphasized the importance of immunotherapy for this disease and opens the possibility of combinatorial approaches.

A phase I study conducted in China has used a CD30.CAR, in which a CD30-specific scFv was combined with a 4-1BB costimulatory endodomain and delivered through a lentiviral vector (25), with the CAR-Ts being infused after conditioning chemotherapy. We previously described and preclinically tested a CD30.CAR that is derived from a different antibody (HSR3), makes use of the CD28 costimulatory endodomain, and is delivered using a gammaretrovirus $(26,27)$. We have now used this CAR in a phase I dose escalation study in patients with relapsed/refractory CD30 ${ }^{+}$ HL and ALCL malignancies and show, for the first time to our knowledge, the induction of complete response (CR) in relapsed/ refractory patients with HL or ALCL even in the absence of conditioning, and without significant toxicities.

\section{Results}

Patient characteristics. We administered CD30.CAR-Ts to 9 patients with $\mathrm{EBV}^{-}, \mathrm{CD} \mathrm{O}^{+}$lymphoid malignancies. Specifically, 6 patients had HL, 1 patient had cutaneous anaplastic lymphoma kinase-negative (ALK) ALCL, 1 patient had $\mathrm{ALK}^{+}$systemic ALCL, and 1 patient had a composite lymphoma (diffuse large $B$ cell lymphoma evolved to HL) (Figure 1). Patient characteristics are summarized in Table 1 . All but 1 patient (patient 7) had active disease at the time of CD30.CAR-T infusion, and all patients had relapsed after 3 or more lines of chemotherapy. None of the patients received lymphodepleting chemotherapy prior to CD30.CAR-T infusion, and 7 patients who had received brentuximab had this drug discontinued more than 1 month before CD30.CAR-T infusion (Table 1). Seven patients for whom autologous CD30.CAR-Ts were manufactured did not receive their cell infusion because: they declined to participate in the infusion part of the study and pursued alternative treatment outside our institution (3 patients); they experienced progressive disease while waiting for their $\mathrm{T}$ cell infusion during the period of safety observation between patients and doses according to protocol (2 patients); or they did not meet the inclusion criteria at the time scheduled for the T cell infusion (2 patients) (Figure 1).

Characteristics of manufactured CD30.CAR-Ts. Twenty-two CD30.CAR-T products were manufactured using either IL-2 (11 products) or IL-7/IL-15 (11 products) within $15 \pm 4$ and $15 \pm 2$ days, respectively (Figure 2 and Supplemental Table 1; supplemental material available online with this article; https://doi.org/10.1172/ JCI94306DS1). By day 15 of culture, CD30.CAR-Ts grown in IL-7/ IL-15 had greater expansion from baseline and higher final cell numbers ( $45 \pm 13$ and $1.2 \times 10^{9} \pm 5.5 \times 10^{8}$, respectively) than those expanded in IL-2 $\left(27.4 \pm 13\right.$ and $6.5 \times 10^{8} \pm 3.3 \times 10^{8}$, respectively; $P=0.019, t$ test on log-transformed data) (Figure 2A). CAR expression was comparable ( $>89 \%$; corresponding to mean transgene copy numbers of 71,140 $\pm 7,800$ per 100 ng of DNA) in all manufactured products (Figure 2B). CD30.CAR-Ts were composed of more than $99 \% \mathrm{CD}^{+} \mathrm{T}$ cells, and each contained a variable ratio of $\mathrm{CD}^{+}$and $\mathrm{CD}^{+} \mathrm{T}$ cells, with an overall $\mathrm{CD}^{+} \mathrm{T}$ cell predominance when grown in IL-2 $(61.6 \% \pm 12.6 \%)$ compared with IL-7/ IL-15 (42.3\% $\pm 18.7 \% ; P=0.01)$ (Figure $2 \mathrm{C})$. The majority of CD30. CAR-Ts were $\mathrm{CD}_{45 \mathrm{RO}^{+}}$and only a small fraction expressed central memory-associated phenotypic markers such as CD62L and CCR7 (Supplemental Table 1), which may reflect the heavy pretreatment of the patients enrolled in our trial (Table 1). Natural killer cells $\left(\mathrm{CD}^{-} \mathrm{CD}^{-} 6^{+}\right)$were not detectable. CD30.CAR-Ts grown in IL-7/IL-15 expressed higher levels of CXCR4 and CXCR3, which are chemokine receptors known to promote $\mathrm{T}$ cell migration to peripheral tissues (Supplemental Table 1). All CD30.CAR-T products were cytotoxic to $\mathrm{CD}^{3} \mathrm{O}^{+}$target tumor cells in vitro (Figure 2D), but had negligible activity against $\mathrm{CD}^{-} 0^{-}$target tumor cells.

CD30.CAR-T expansion and persistence. We gave CD30.CARTs to each patient as a single administration over a 2- to 5-minute period. Molecular signals (genomic quantitative PCR [qPCR]) for CD30.CAR-Ts were detected in the peripheral blood of all patients by 3 hours after infusion ( $94 \pm 23$ copies/ $\mu$ g of peripheral blood mononuclear cell [PBMC] DNA), and these signals increased and peaked within the 1 week after infusion in a dose-dependent manner, with the highest detection at the third dose level $(5,791 \pm 2,463$ 


\section{Table 1. Patient characteristics}

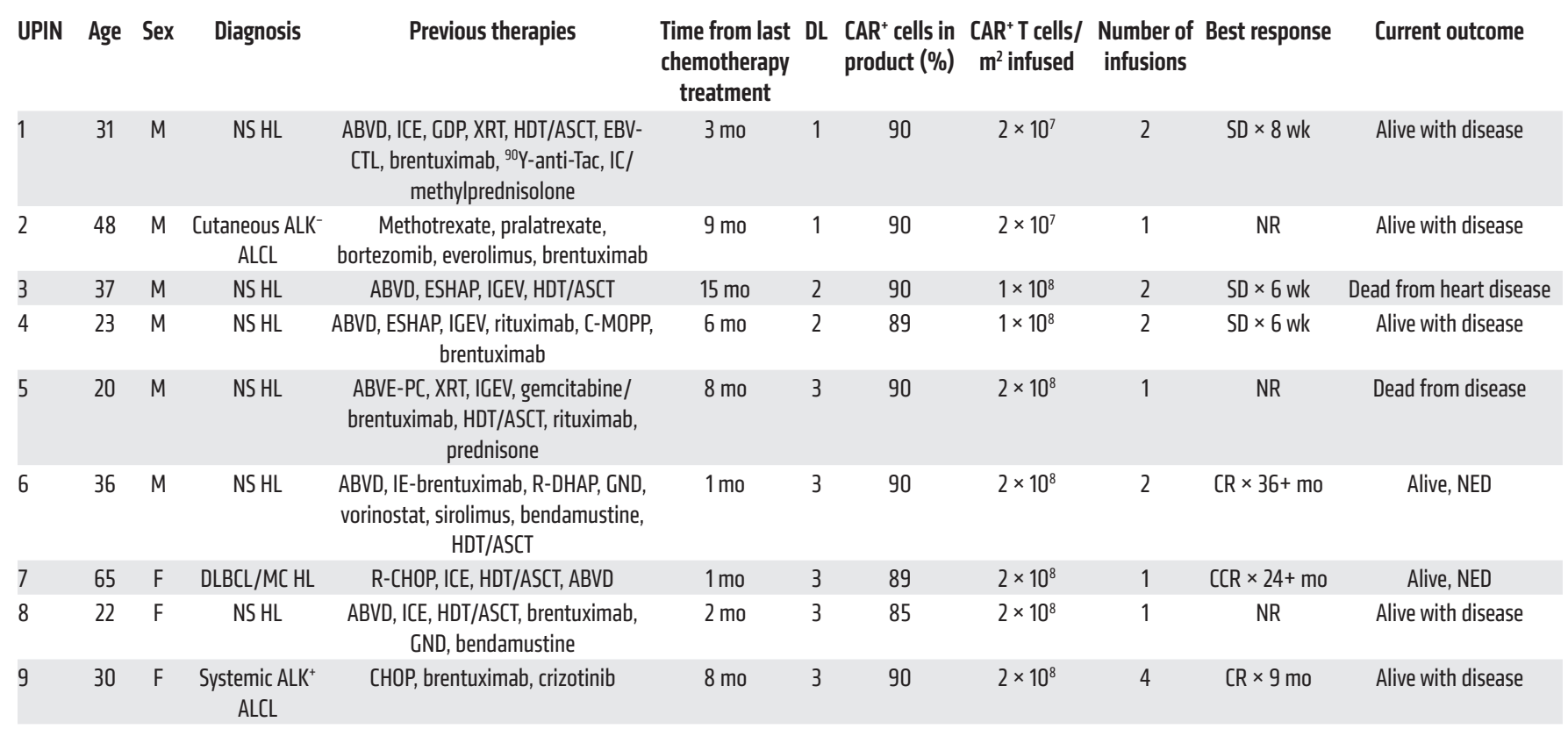

ABVD, doxorubicin, bleomycin, vinblastine, dacarbazine; ABVE-PC, doxorubicin, bleomycin, vincristine, etoposide, prednisone, cyclophosphamide; CCR, continued complete response; (R)-CHOP, (rituximab), cyclophosphamide, doxorubicin, vincristine, prednisone; C-MOPP, cyclophosphamide, vincristine, procarbazine, prednisone; CR, complete response; DL, dose level; DLBCL, diffuse large B cell lymphoma; EBV-CTL, EBV-specific cytotoxic T lymphocytes; ESHAP, etoposide, methylprednisolone, cytarabine, cisplatin; GDP, gemcitabine, dexamethasone, cisplatin; GND, gemcitabine, navelbine, liposomal doxorubicin; HDT/ASCT, high-dose therapy followed by autologous stem cell transplant; I(C)(E), ifosfamide, (carboplatin), (etoposide); IGEV, ifosfamide, gemcitabine, vinorelbine; MC HL, mixed-cellularity HL; NED, no evidence of disease; NR, no response; NS HL, nodular sclerosis HL; R-DHAP, rituximab, dexamethasone, cytarabine, cisplatin; SD, stable disease; UPIN, universal patient identifier number; XRT, radiation therapy.

copies/ $\mu \mathrm{g}$ of PBMC DNA) (50-fold increase compared with 3 hours after infusion, ranging from 7 to 158) (Figure 3A). Molecular signals then declined over the ensuing weeks (106 \pm 41 copies $/ \mu \mathrm{g}$ of DNA 3 weeks after infusion), though remaining detectable for more than 6 months after infusion in 6 patients (Supplemental Figure 1A). Seven patients received a second infusion of CD30.CARTs, and 1 patient received a total of 4 infusions, which produced only modest expansion of CD30.CAR-Ts in the peripheral blood (Supplemental Figure 1, A and B). Molecular signals were below the threshold needed to detect distinct CAR-Ts by flow cytometry in patients treated at the first and second dose levels, but CD30. CAR-Ts were consistently detectable in the peripheral blood in patients at the third dose level (Figure 3B and Supplemental Figure 1A). A statistical correlation was observed between CD30.CAR-T expansion and dose level $(P=0.002$, Pearson correlation; Figure $3 \mathrm{C}$ ) and the frequency of $\mathrm{CD} 4^{+} \mathrm{T}$ cells in the infused product $(P=0.047$; Supplemental Figure 1, C and D; the correlation was not statistically significant for CD8 cells, $P=0.09$ ), while there was no correlation with preinfusion absolute lymphocyte cell and white blood cell counts, disease type, or cytokines used to manufacture CD30.CAR-Ts. Notably, we only infused 3 patients whose product was generated with IL-7 and IL-15 (patients 7, 8, and 9). Responses were observed in 2 of them. Interestingly, the patient whose disease did not respond had undetectable CD45RA ${ }^{+}$cells, while overall the 3 responding patients had the highest percentage of CD45RA ${ }^{+} \mathrm{CD}^{+}$cells (Supplemental Figure $1 \mathrm{~F}$ ).
Acute and long-term toxicities. CD30.CAR-T infusions were well tolerated at all dose levels and after 1 or more infusions (Table 1). None of the adverse events reported (most frequently fatigue, hyper- or hypokalemia, and transient elevation of aspartate aminotransferase [AST]) were considered related to CD30. CAR-T infusion. After infusion, no significant differences were observed in white blood cell counts (Supplemental Figure 2, A and B) apart from modest reduction in eosinophils (Figure 4A). B and $\mathrm{T}$ cell counts remained stable (Supplemental Figure 3, A and B). No patients developed symptoms consistent with cytokine release syndrome. Elevations of inflammatory cytokines, such as IL-6 and TNF- $\alpha$, were observed, which generally coincided with the peak of CD30.CAR-T expansion, but the fold increases from baseline were modest (Figure 4B and Supplemental Figure 2C). Similarly, no significant changes were observed in plasma levels of IL-15, IL-7, or IL-2. As CD30 is expressed on activated T cells (28), we monitored $\mathrm{T}$ cell immunity to viral antigens in all patients before and after infusion of CD30.CAR-Ts. Confirming our preclinical assessment (26), CD30.CAR-Ts did not impair the frequency of T cells (CTLp) reactive to common viral pathogens such as EBV, CMV, adenovirus, or influenza (Figure 4C). No patients developed viral infections after receiving CD30.CAR-Ts.

Clinical outcomes and correlative studies. We observed clinical responses in 3 of 9 patients ( $2 \mathrm{CR}, 1$ continued CR), as well as 3 patients with stable disease (Figure 5A). Patient 9 (ALK ${ }^{+}$ALCL) had a dramatic response after the first CD30.CAR-T infusion (Figure 5B) 
A

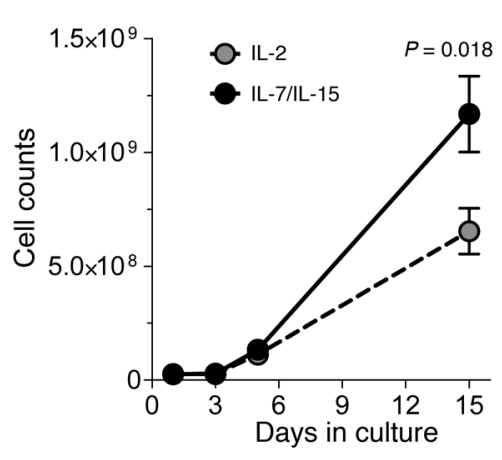

B

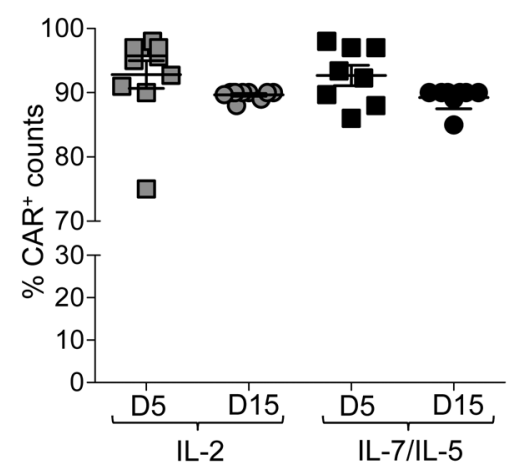

C

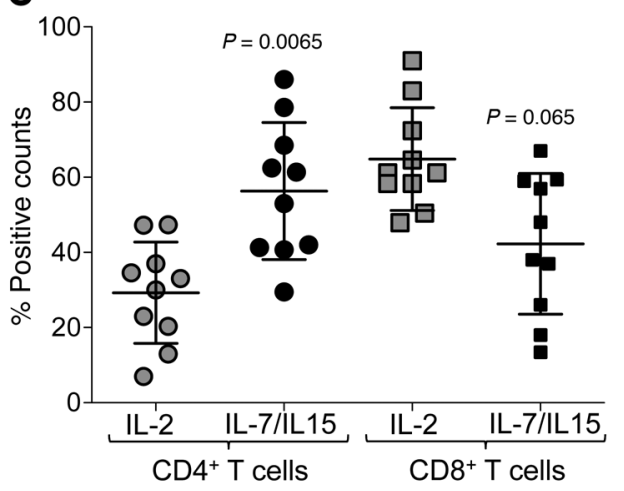

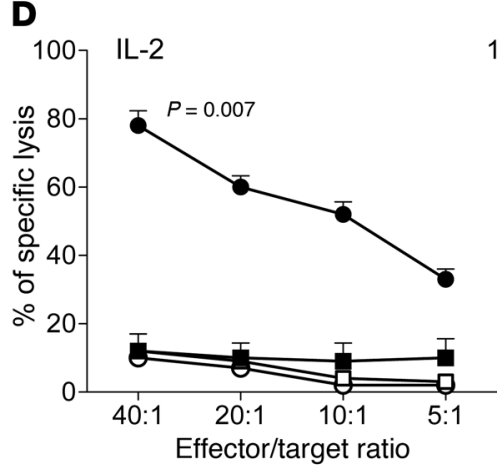

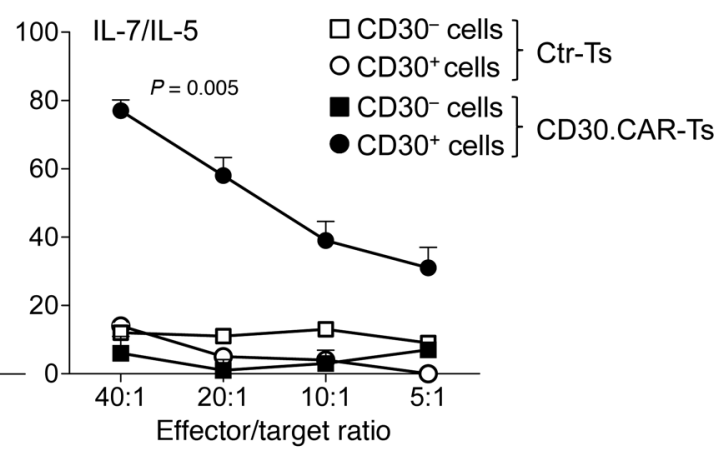

Figure 2. Characteristics of CD30.CAR-Ts expanded in IL-2 or IL-7/IL-15. (A) Total cell number at the time of transduction and clinical freeze of CD30.CAR-T products ( $n=11$, unpaired $t$ test) grown in IL-2 (gray circles) or in IL-7/IL-15 (black circles). (B) Percentage of CAR ${ }^{+}$T cells upon removal from retronectincoated plates (day 5, squares) and at the time of freezing (day 15, circles), grown in IL-2 (gray) or in IL-7/IL-15 (black). Data in $\mathbf{A}$ and $\mathbf{B}$ are mean \pm SEM. (C) Percentage of $\mathrm{CD}^{+}$(circles) or CD8 ${ }^{+}$(squares) T cells when grown in IL-2 (gray) or in IL-7/IL-15 (black), at the time of clinical freeze. Data are mean \pm SD ( $n=10$, unpaired $t$ test). (D) Cytotoxic activity of CD30.CAR-Ts (black symbols) or nontransduced, control (Ctr) T cells (white symbols) expanded in IL-2 (left graph, $n=9$; paired $t$ test) or in IL-7/IL-15 (right graph, $n=8$; paired $t$ test). Targets were CD30+ tumor cells (HDLM-2, squares) or CD30- tumor cells (Raji, circles). Data are shown as mean \pm SEM for all the generated products.

and was deemed to achieve a CR after the fourth infusion that was maintained for 9 months. Before CD30.CAR-Ts, patient 6 had residual pelvic lymphadenopathy (standardized uptake value of 3.3, which was above the activity in the mediastinum blood pool) after high-dose therapy and autologous stem cell transplantation. Six weeks after the first infusion of CD30.CAR-Ts, a repeat PET scan showed resolution (no activity above mediastinum blood pool), which was also documented in a scan performed 20 weeks later; he has been in CR for more than $2^{1 / 2}$ years (Figure $5 C$ ). Patient 7 had CD30.CAR-T infusion while in CR after salvage chemotherapy given for relapse after autologous stem cell transplantation, and she has remained in continued complete response for more than 2 years. Patients 1, 3, and 4 (nodular sclerosis HL) had transient stable disease at the 6-week evaluations. The remaining 3 patients had progressive disease (Figure 5A). Objective clinical responses were mainly achieved in patients infused at the third dose level.

Although the 7 patients who received 2 or more CD30.CAR-T infusions had lower CAR-T expansion following the second or subsequent infusion than after the initial cell dose (Supplemental Figure 1, A and B), human anti-mouse antibody (HAMA) remained nondetectable or below levels of biological activity (maximum level 12.5 U/ $\mathrm{ml}$, with a negative HAMA assay defined as $\leq 74 \mathrm{U} / \mathrm{ml}$ ) (Figure 6A), even after multiple infusions. The amount of peripheral blood we were permitted to collect after CD30.CAR-T infusions was insuffi- cient to directly explore the emergence of cellular immune responses to the CD30.CAR in vivo. However, we consistently failed to elicit cytotoxic $\mathrm{T}$ cell responses targeting the CD30.CAR by exposing healthy individual $\mathrm{T}$ cells to multiple rounds of CAR.CD30-Tloaded T cells in vitro (ref. 29 and Supplemental Figure 4). The frequency of $\mathrm{CD}^{+}{ }^{+} \mathrm{FoxP}^{+}$regulatory $\mathrm{T}$ cells (Tregs) in the peripheral blood was $7.2 \% \pm 1.3 \%$ before infusion and did not significantly change after CD30.CAR-T infusions $(6.3 \% \pm 1.4 \%$ and $6.4 \% \pm 0.9 \%$ 1 week and 2 weeks after infusion, respectively) (Figure 6B). No correlation was found between the frequency of Tregs in the peripheral blood, expansion and persistence of CD30.CAR-Ts, or clinical response (data not shown). However, the limited expansion of CARTs in the circulation after subsequent infusions, combined with the generally poorer persistence with subsequent infusions, supports the possible development of an unidentified negative factor in the host.

Soluble CD30 (sCD30) is typically elevated in advanced HL (30). Although CD30.CAR-Ts are not blocked by $\operatorname{SCD} 30(26,31)$, we found an inverse correlation $(P=0.0087)$ between sCD30 levels at the time of CD30.CAR-T infusion and the subsequent expansion of CD30.CAR-Ts (Figure 6C). Notably, in responding patients the peak of CD30.CAR-T molecular signals in peripheral blood coincided with a transient increase of $\mathrm{SCD} 30$ consistent with the release of SCD30 upon tumor destruction (ref. 30, Figure 6D, and Supplemental Figure $3 \mathrm{C}$ ). Finally, numerous reports have described high expression 
A
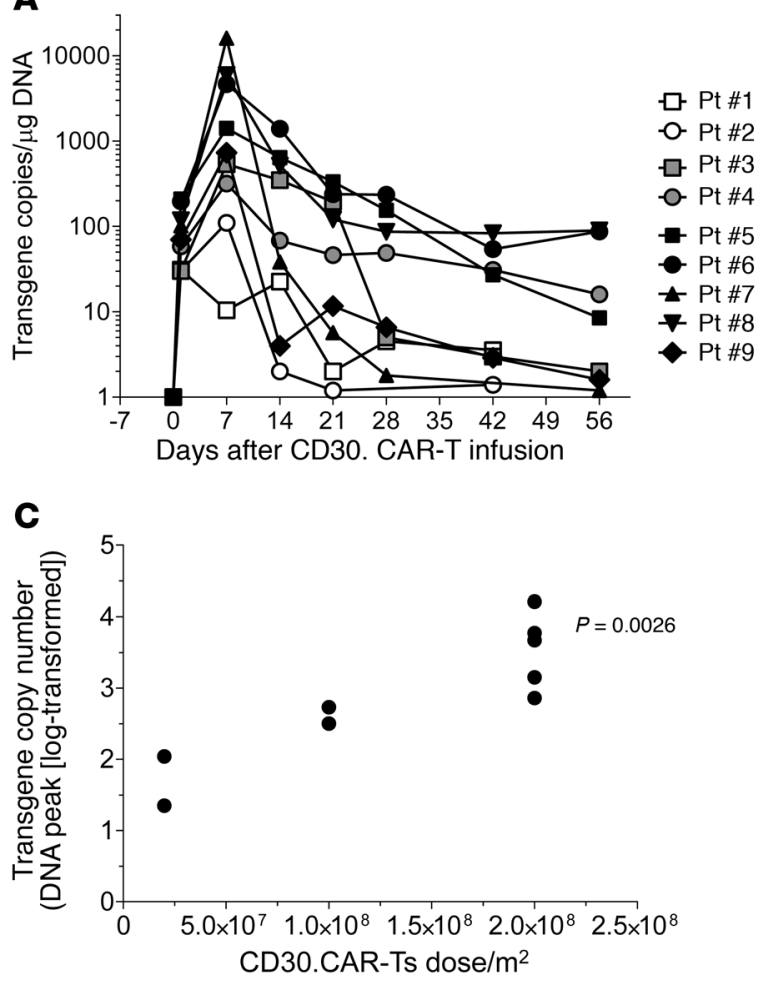

B

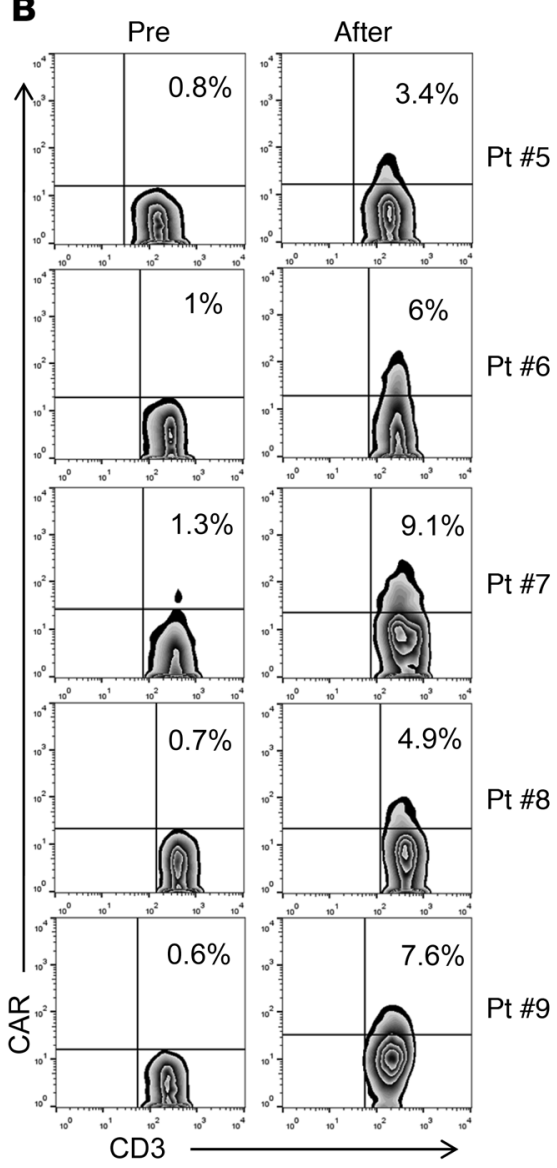

Figure 3. In vivo expansion and persistence of infused CD30.CAR-Ts as assessed by qPCR in the peripheral blood. (A) Detection of CD30.CAR-T molecular signals by qPCR. Data points represent critical postinfusion intervals after the first infusion of CD30.CAR-Ts. Lines denote each patient (legend shows universal patient identifier numbers [UPINs]). White symbols are from patients on dose level 1, gray symbols are from patients on dose level 2, and black symbols are from patients on dose level 3. (B) Detection of CD30.CAR-Ts in the peripheral blood using flow cytometry for patients treated at dose level 3. Cells were gated on T lymphocytes identified as $\mathrm{CD}_{4} 5^{+}$and $\mathrm{CD}^{+}$cells. Shown are zebra plots before and 1 week after infusion. (C) Pearson correlation between the cell dose of CD30.CAR-T and the peak of the CD30.CAR-T molecular signals (log transformed) for the 9 infused patients.

of PD-L1 by Reed-Sternberg cells (23), and infusion of blocking antibodies to PD1 has produced significant clinical responses in patients with the disease (22-24). We therefore evaluated the expression of the PD-L1 cognate receptor PD1 in infused CD30.CAR-Ts. Overall, PD1 was expressed by $33 \% \pm 9 \%$ of infused CD30.CAR-Ts (Figure $6 \mathrm{E})$, but no correlation was observed between PD1 expression and CD30.CAR-T expansion/persistence in vivo.

\section{Discussion}

We report here the outcome of 9 patients with relapsed/refractory EBV-negative HL and ALCL treated with autologous CD30.CARTs in a dose-finding study in which $\mathrm{T}$ cells were infused in patients without a conditioning regimen. Promising antitumor activity was documented in both HL and ALCL with the achievement of CR.

CD30 is an attractive target for immunotherapy because it is highly expressed by Hodgkin Reed-Sternberg (HRS) cells and by the malignant cells of ALCL. However, CD3O is also transiently expressed by a small fraction of activated B and T cells (28), and thus concerns remain that effective CD30.CAR-Ts could further impair cell-mediated immunity in patients who are already immunodeficient. None of our patients experienced significant changes in $\mathrm{T}$ or B cell counts, or impairment in the frequency of circulating virus-specific $\mathrm{T}$ cells, and infection episodes were not observed, indicating an overall good safety profile of CD30.CAR-Ts. A fraction of eosinophils has been reported to express CD30 (28), and we observed a transient decrease of these cells in the peripheral blood after CD30.CAR-T infusions. Since eosinophils may promote the growth of HRS cells, a similar reduction of these cells at the tumor site may be beneficial (32).

In our study, CD30.CAR-Ts were well tolerated, and CRs were observed at the highest dose of CD30.CAR-Ts and after 2 or more $\mathrm{T}$ cell doses. The highest degree of expansion of circulating CD30. CAR-Ts (area under the curve, Supplemental Figure 1E) occurred at the third dose level. Our study has the major strength of showing direct effects of CD30.CAR-Ts, in contrast to results from Wang et al. (25), who followed the more general practice of using lymphodepletion prior to CAR-T infusion (2, 3, 5, 6, 9, 13, 33-35). There are several other differences between our trial and the one described by Wang et al., including the method for transgene delivery (lentiviral versus $\gamma$-retroviral) and the costimulatory endodomains (4-1BB versus CD28). Nonetheless, both lentiviral and $\gamma$-retroviral vectors, and 4-1BB and CD28 endodomains have been associated 
A
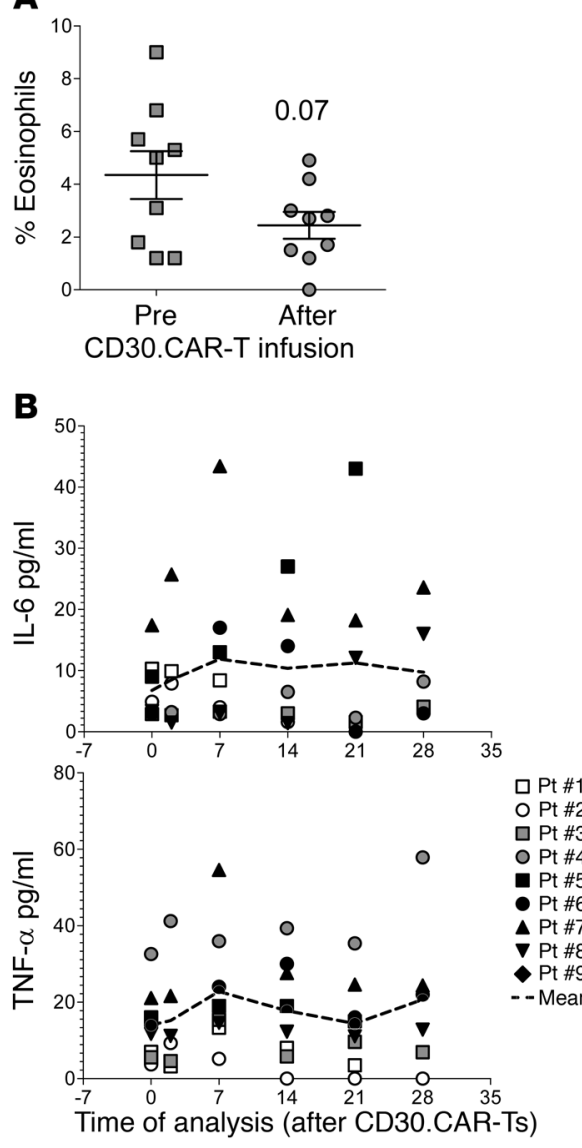

C
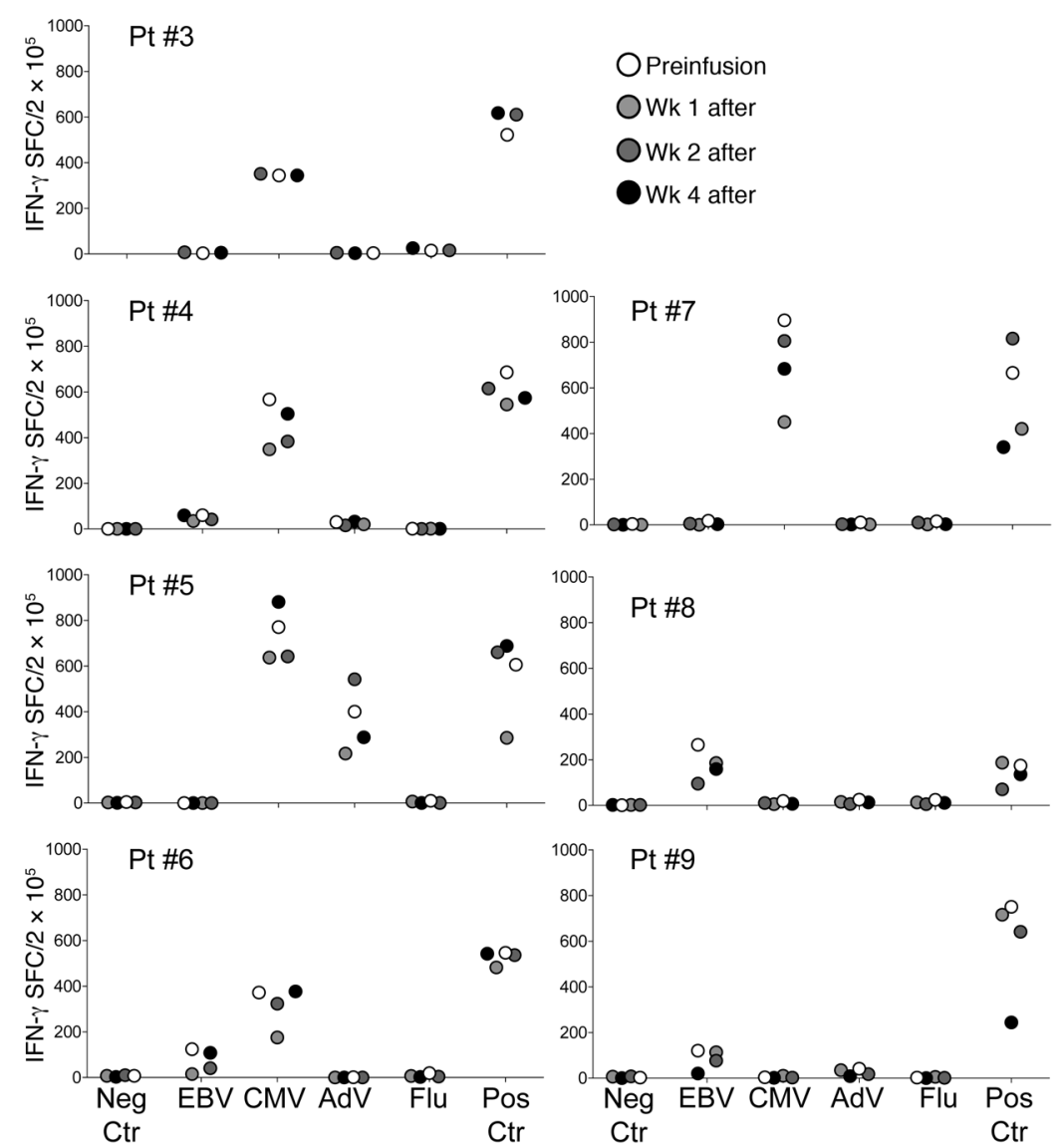

Figure 4. Immunological effects of infused CD30.CAR-Ts. (A) Decrease of circulating eosinophils in the peripheral blood after CD30.CAR-T infusions. Shown are means \pm SEM ( $n=9$; paired $t$ test). (B) Plasma levels of inflammatory cytokines (shown are IL-6 and TNF- $\alpha$ ) modestly increased after CD30. CAR-T infusion. Each symbol denotes a different patient (legend shows universal patient identifier numbers [UPINs]), and the dashed line represents the mean value. (C) Frequency of T cells targeting viral antigens in the peripheral blood at different time points before and after CD30.CAR-T infusions as assessed by IFN- $\gamma$ ELISPOT assays. SFCs, spot-forming cells.

with responses using other CARs (2-9, 13, 33-35). Therefore, these structural differences may not themselves account for the observed differences in response. An alternative possibility is that the affinity of the $\mathrm{scFv}$ may play a role, since $\mathrm{sCD} 30$ is present in the plasma of patients with advanced/aggressive disease. While the scFv we used in our CAR was previously shown to be unaffected by SCD30 $(26,31)$, no information is available for the scFv used by Wang et al. Moreover, we observed our best responses in patients with low sCD30, suggesting that a lower burden of disease may be important. No sCD30 data are available for patients in the Wang et al. trial.

The correlation between the dose of CD30.CAR-Ts and the in vivo expansion we observed in this small cohort of patients suggests that host lymphodepletion before CAR-T infusion would be beneficial in further improving their expansion and their antitumor activity. Indeed, while CD19-specific CAR-Ts encounter B cell targets in the peripheral blood upon infusion and thus receive immediate and appropriate costimulation, the expansion of CD30.CAR-Ts in the absence of circulating target cells may require homeostatic cytokines like IL-15 that will be more available following lymphodepletion (36). At present, the optimal conditioning regimen is unknown (25) and needs to balance the requirement for sufficient lymphodepletion and disruption of the tumor environment, with the desire to prevent toxicity from the depletion regimen itself, or the subsequently greater CAR-T expansion. However, chemotherapy may also prove dispensable for $\mathrm{HL}$, a disease subset characterized by intrinsic overall immune deficiency (37). Indeed, previous experience with adoptive $\mathrm{T}$ cell therapy in EBV-associated HL has shown that responses can be achieved with the adoptive transfer of EBV or LMP-CTLs even in the absence of chemotherapy conditioning $(20,21,38)$. Finally, we cannot rule out the possibility that the incorporation of IL-7 and IL-15 in the manufacturing procedure contributes to improved CAR-T functions.

Although CD30.CAR-Ts expanded in peripheral blood, their persistence was brief. Even after 2 or more CD30.CAR-T infusions we detected no changes in HAMA in the peripheral blood, and thus interference with CD30.CAR-Ts by CAR antibodies seems unlikely. Unfortunately, we did not have sufficient material to perform in-depth analysis of T cell responses against potential "epitopes" within the $\operatorname{CAR}(33,39)$, so we cannot formally exclude the presence of $\mathrm{T}$ cells targeting CAR-derived epitopes. However, our in vitro analysis using a methodology previously used to successfully generate ex vivo $T$ cell responses against extremely weak immunogenic antigens 
A

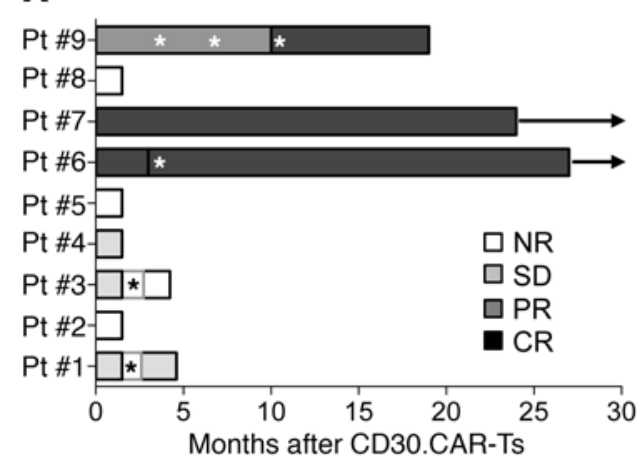

B

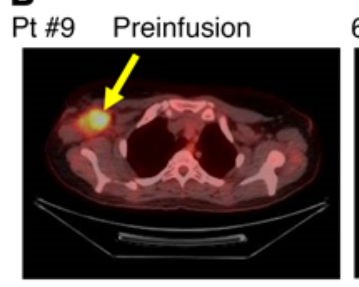

6 wks after CD30.CAR-Ts

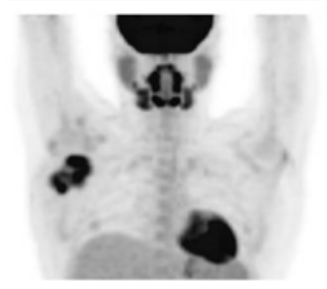

C
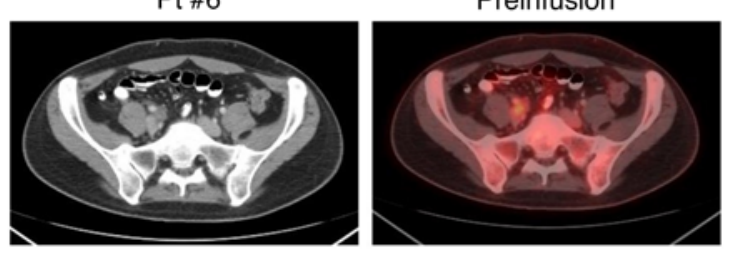

20 wks after CD30.CAR-Ts

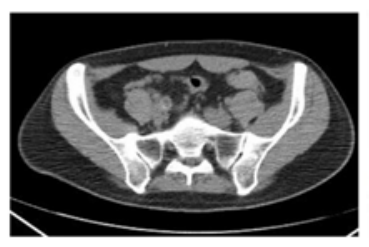

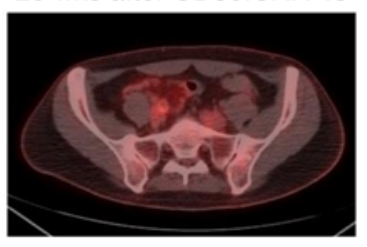
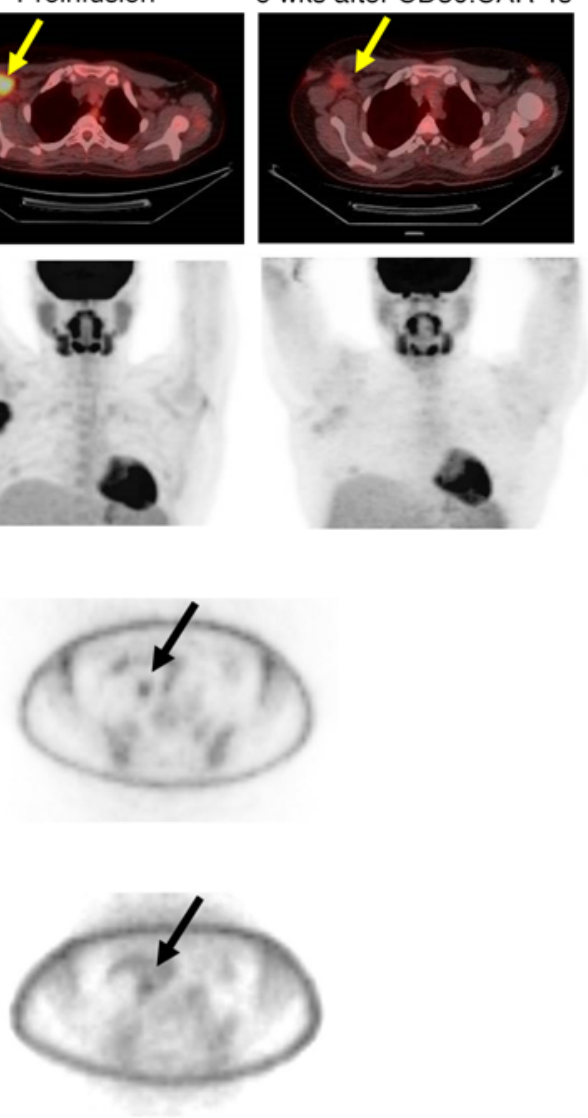

Figure 5. Antitumor effects of CD30.CAR-Ts. (A) Clinical responses for the 9 infused patients. Arrows indicate ongoing remission. NR, no response; SD, stable disease; PR, partial response; CR, complete response. Asterisks indicate subsequent infusions. (B) Patient 9 had ALK+ ALCL with active disease in the right axillary, as documented in the preinfusion PET/ CT scan. Six weeks after the first infusion of CD30.CAR-Ts, PET showed PR. Another PET scan obtained after a fourth infusion was consistent with CR. This patient was without evidence of disease for 9 months. (C) Patient 6 had a residual pelvic lymphadenopathy (standardized uptake value of 3.3 , which was above the activity in the mediastinum blood pool) after high-dose therapy and autologous stem cell transplantation. Six weeks after the first infusion of CD30.CARTs, a repeat PET scan showed resolution (no activity above mediastinum blood pool), which was also documented in a scan performed 20 weeks later.
(29) failed to elicit any T cell responses against the CD30.CAR. The limited persistence of CD30.CAR-Ts may also be a consequence of the affinity of the CD30-specific scFv used to construct the CD30. CAR. CD3O is present in a soluble form in the plasma of HL patients with advanced/aggressive disease (30), and the affinity of scFv may prove critical in determining the overall avidity of the CD30.CAR-Ts for sCD30 as opposed to membrane-bound CD30. Extensive binding of sCD30 may render CD30.CAR-Ts more susceptible to sequestration by scavenger cells. Although the $\mathrm{scFv}$ derived from the HSR3 antibody used to generate our CD30.CAR has been previously shown to be unaffected by $\operatorname{sCD} 30(26,31)$, we found an inverse correlation between sCD30 levels at the time of the CD30.CAR-T infusion and expansion of CD30.CAR-Ts. Finally, the specific design of the CD30. CAR we used may affect their persistence. Our CD30.CAR construct contains a long IgG1 spacer region that may bind to the Fc receptor of monocytes/macrophages, and also favor CD30.CAR-T sequestration (40). In view of the clinical responses achieved, it seems unlikely that these cells were totally eliminated, and the disappearance of CD30.CAR-T molecular signals may instead reflect relocalization of CD30.CAR-Ts in peripheral tissues (25).

HRS cells often express high levels of PD-L1, and checkpoint blockade with nivolumab or pembrolizumab shows significant efficacy in HL (22-24). Indeed, nivolumab has been granted accelerated FDA approval for treatment of HL progressing after treatment with brentuximab vedotin and autologous stem cell transplant. Nevertheless, checkpoint blockade is expected to be ineffective in the absence of endogenous tumor-infiltrating lym- phocytes recognizing tumor neoantigens, or in the event of MHC dysfunction (41). In these patients the adoptive transfer of CD30. CAR-Ts opens the opportunity to provide tumor-specific $\mathrm{T}$ cells and thereby increase the effectiveness of checkpoint blockade.

In summary, our study demonstrates the tolerability, safety, and potential efficacy of CD30.CAR-Ts in CD30+ lymphoid malignancies. Appropriate tumor reduction and lymphodepletion before CD30.CAR-T infusion should enhance their clinical activity without increasing toxicity. Future exploration of the synergy between CD30.CAR-Ts and PD1/PD-L1 blockade may also prove beneficial.

\section{Methods}

Clinical study. We conducted a phase I study (NCT01316146) designed to assess the feasibility and safety of infusing escalating doses of autologous, polyclonally activated, peripheral blood $\mathrm{T}$ cells that were genetically modified to express a CD30-specific CAR (CD30.CAR) (31) encoding the $\mathrm{CD} 28$ endodomain in patients with relapsed/refractory $\mathrm{CD} 3 \mathrm{O}^{+}$ lymphoproliferative disorders (HL and non-Hodgkin lymphoma). All patients with a diagnosis of HL had CD30 positivity determined by a pathologist according to her/his institutional hematopathology standard measured against positive and negative controls. In all those cases, the Reed-Sternberg cells were deemed $\mathrm{CD}_{3} \mathrm{O}^{+}$and $\mathrm{CD} 15^{+}$(in a membrane/ Golgi pattern, when described). Patient 2 (ALK ${ }^{-}$ALCL) had malignant cells that were also reported by a pathologist to be positive for CD30, CD4, and epithelial membrane antigen (EMA). Patient 9 (ALK ${ }^{+}$ALCL), who had the best response, had malignant cells that were described as having "strong membranous and cytoplasmic staining with Golgi accen- 
A

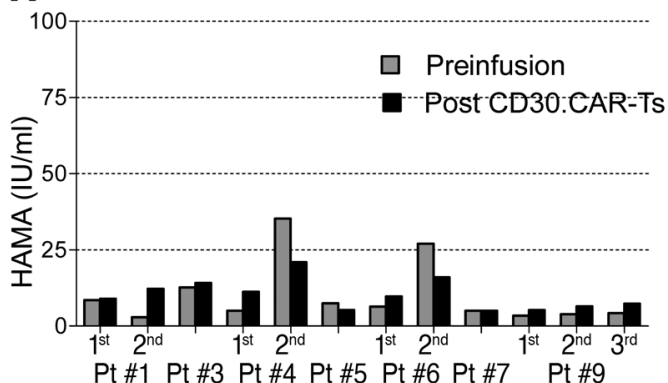

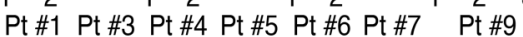

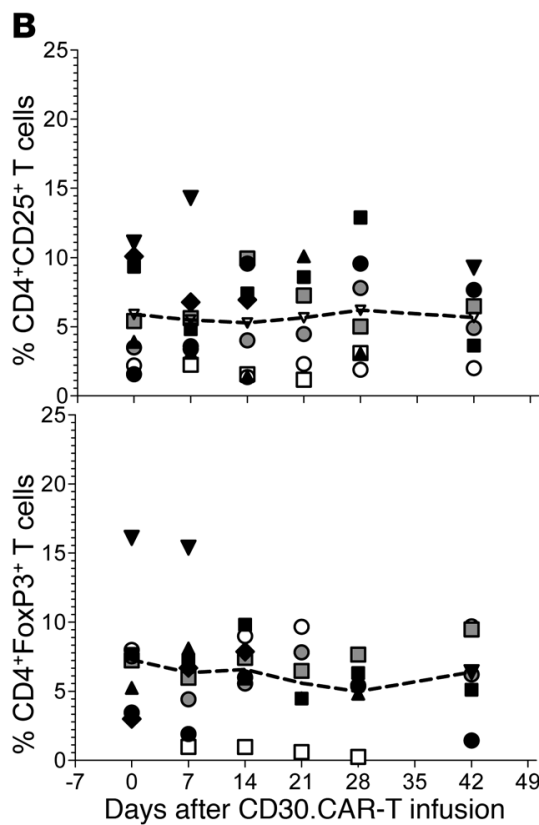

C

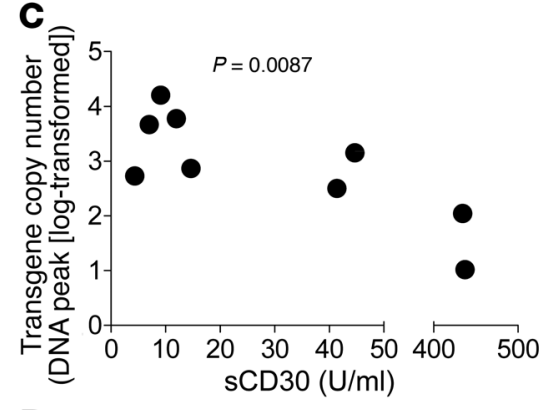

D

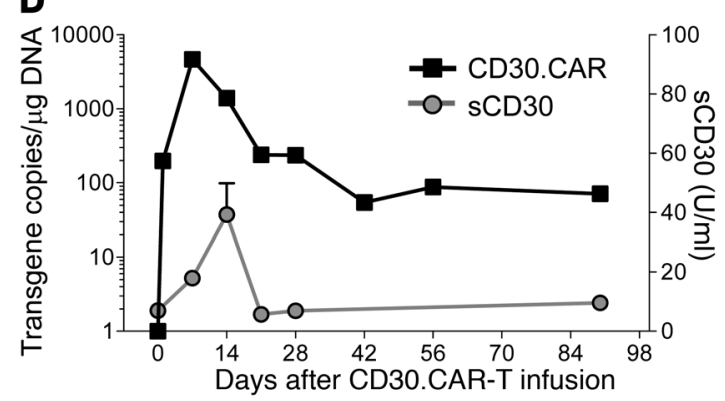

E

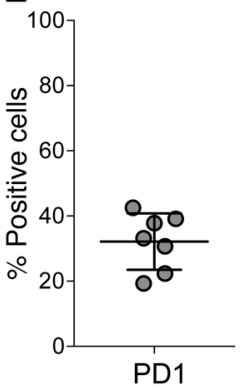

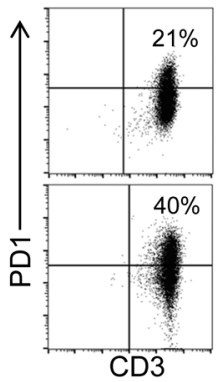

Figure 6. Correlative biological studies after CD30. CAR-Ts. (A) There was no significant generation of human anti-mouse antibody (HAMA) after CD30.CAR-T infusions. (B) Shown are percentages of $\mathrm{CD} 4^{+} \mathrm{CD} 25^{+}$ (top graph) and $\mathrm{CD}^{+} \mathrm{FoxP3}^{+}$ (bottom graph) T cells for the 9 infused patients. Each symbol denotes a different patient (UPINs), and the dashed line indicates the mean values. (C) Pearson correlation between peak of CD30.CAR-T molecular signals (log transformed) and sCD30 cell preinfusion for the 9 infused patients. (D) CD30.CAR-T molecular signals and sCD30 levels in the peripheral blood for patient 6. (E) The graph shows the percentage of $\mathrm{PD1}{ }^{+} \mathrm{CD} 30$. CAR-Ts in the infused products (shown are means $\pm \mathrm{SD} ; n=7)$. On the right, dot plots from 2 representative patients are shown. tuation" for CD30. No chemotherapy was given to patients immediately before or after infusion of CD30.CAR-transduced T cells (CD30.CARTs). We administered 3 dose levels of CD30.CAR-Ts, $2 \times 10^{7}$ (dose level 1), $1 \times 10^{8}$ (dose level 2), and $2 \times 10^{8}$ (dose level 3 ) cells $/ \mathrm{m}^{2}$, based on $\mathrm{CAR}^{+}$ $\mathrm{T}$ cells. CD30.CAR-Ts were always cryopreserved and administered after thawing. Infusion of CD30.CAR-Ts occurred over a 2- to 5-minute period. We used an interpatient dose escalation that followed a continual reassessment method, which required safety to be demonstrated 6 weeks after infusion in 2 patients at each dose level before escalation. Additional infusions of the same dose of CD30.CAR-Ts were allowed as long as there was evidence of clinical benefit, defined as at least stable disease. Additional infusions were administered at least 6 weeks apart without intervening chemotherapy. Clinical and laboratory evaluations were performed at weeks 1, 2, 4, and 6 and months 3, 6, 9, and 12 after CD30.CAR-T infusion, and yearly thereafter. Adverse events during and after $\mathrm{T}$ cell infusions were graded according to the NIH Common Terminology Criteria for Adverse Events, version 4 (http://ctep.cancer. gov). Responses were assessed by imaging at week 6 after CD30.CAR-T infusion and were defined as complete response (CR), partial response, stable disease, or progressive disease, according to the National Cancer Institute's International Working Group criteria (42).

Generation of the retroviral construct. The scFv of the CD30.CAR is derived from a murine antibody and has not been humanized. This construct consists of the $\mathrm{CD} 30$-scFv fragment (31) cloned in frame with the sequence encoding the human IgG1.CH2- $\mathrm{CH} 3$ domains, the transmem- brane and cytoplasmic domain of the CD28 costimulatory endodomain, and the $\zeta$ chain. The CAR cassette was then subcloned into the SFG retroviral backbone (26). We generated the clinical-grade packaging cell line using PG13 cells (gibbon ape leukemia virus pseudotyping packaging cell line; American Type Culture Collection CRL-10686) (7). We used the highest-titer clone to establish a master cell bank and released the clone for clinical use only after safety testing and vector sequencing. We stored the final viral supernatant at $-80^{\circ} \mathrm{C}$ and tested it before clinical release.

Generation and transduction of CD30.CAR-Ts. To generate CD30. CAR-Ts, we transduced PBMCs obtained by Ficoll density centrifugation of peripheral blood obtained by conventional phlebotomy 1-3 months before CD30.CAR-T administration. We activated PBMCs with OKT3 antibody (Janssen Biotech) or CD3 and CD28 antibodies (Miltenyi Biotec), and recombinant human IL-2 (IL-2, 100 U/ml; Prometheus), or IL-7 (10 ng/ml; R\&D Systems) and IL-15 (5 ng/ml, R\&D Systems), in 24-well plates precoated with a recombinant fibronectin fragment (FN CH-296, Retronectin Takara; Clontech). After transduction, T cells were expanded ex vivo in the presence of IL-2 (100 U/ml) or IL-7 (10 ng/ml) and IL-15 (5 ng/ml), added twice a week. The change in the manufacture process with IL-7 and IL-15 replacing IL-2 took place during the trial (43). The characteristics of the T cell products are summarized in Figure 2 and Supplemental Table 1. Some patients had T cell lines made during the change in manufacturing and had both products available. Nontransduced, control T cells were grown in parallel from each patient in our manufacturing facility, starting from the same 
PBMC product. Generation of CAR-Ts was successfully completed for all PBMCs in which the process was initiated. Products were generated from stored PBMCs, after collection from consented patients.

Immunophenotyping. We used phycoerythrin-, fluorescein isothiocyanate-, peridinin chlorophyll protein-, allophycocyanin-, Alexa Fluor 750-, Alexa Fluor 700-, phycoerythrin cyanin 7-, Pacific Blue-, or KromeOrange-conjugated CD3 (cloneSK7), CD4 (SK3), CD8 (SK1), CD56 (B159), CD19 (SJ25C1), TCR- $\alpha \beta$ (T10B9), TCR- $\gamma \delta$ (B1), CD62L (DREG-56), CD27 (M-T271), CD28 (CD28.2), CD45RA (HI100 or 2H4), CD45RO (UCHL1), CCR2 (clone 48607), CCR4 (1GI), CCR5 (2D7), CCR7 (3D12),CXCR3 (1C6), CXCR4 (12G5), and PD1 (MIH4) (Becton Dickinson/Pharmingen or Beckman Coulter) antibodies to stain the $\mathrm{T}$ cell products. We included control samples labeled with appropriate isotype antibodies and used a "fluorescence minus one" strategy for multicolor staining. We detected the CD30.CAR-Ts with an Fc-specific cyanine-Cy5-conjugated (Fc-Cy5) monoclonal antibody (catalog 109-606-088; Jackson ImmunoResearch), which recognizes the IgG1.CH2-CH3 component of the CAR. We analyzed the cells by FACScan equipped with a filter set for 4 fluorescence signals, using CellQuest software, or a FACS-Canto II, using DIVA software (Becton Dickinson). In some cases cells were collected using the Gallios Flow Cytometer (Beckman Coulter) and analyzed using FlowJo software version 9.3 (Tree Star). Data analysis was performed using FlowJo Software (Tree Star) or Kaluza software (Beckman Coulter).

Cytotoxicity assays. The cytotoxic specificity of each CD30.CAR-T cell line was measured in a standard 4 -hour ${ }^{51} \mathrm{Cr}$ release assay, using effector-to-target ratios of 40:1, 20:1, 10:1, and 5:1. As target cells, we used HDLM-2 (CD30+ tumor cells) and Raji (CD30- tumor cells). The target cells were labeled simultaneously for 1 hour with ${ }^{51} \mathrm{Cr}$. We calculated the percentage of specific lysis as [(experimental release - spontaneous release) $/($ maximum release - spontaneous release $)] \times 100$.

Real-time qPCR of CD30.CAR transgene. We quantified the integrated genome of the retrovirus encoding the CD30.CAR by real-time qPCR. After extracting DNA from peripheral blood samples with the QIAamp DNA Blood Mini Kit (Qiagen) following the manufacturer's instructions, we amplified the DNA with primers and probes (Applied Biosystems) complementary to specific sequences within the retroviral vector. The standard curve was established using serial dilutions of the plasmid encoding the transgene. We performed amplifications using the ABI7900HF Real-Time PCR System (Applied Biosystems) according to the manufacturer's instructions.

ELISA, cytometric bead array, and multiplex assays. We analyzed plasma or serum samples collected before and after CD30.CAR-T infusion using a BD cytokine cytometric bead array (CBA) kit (Becton Dickinson/Pharmingen) or a Milliplex kit (EMD Millipore), following the manufacturers' instructions. In parallel with the samples, we used the human cytokine standards provided with the kit to prepare standard curves. We ran the CBA assays using the FACSCalibur (Becton Dickinson), and analyzed data from the Milliplex kits using the Luminex 200 System and Milliplex Analyst Software (EMD Millipore). We detected sCD30 (eBioscience), TARC (R\&D Systems), and HAMA (Alpha Diagnostic) using specific ELISA and following the manufacturers' instructions.

ELISPOT assays. We used IFN- $\gamma$ ELISPOTs to identify T cells specific for known viral-associated antigens. PBMCs were plated at $2 \times 10^{5}$ to $4 \times 10^{5}$ per well in triplicate in PI-Multiscreen plates (EMD Millipore), and IFN- $\gamma$ production was measured in response to stimulation with peptide mixtures consisting of 15-mer peptides overlapping by 11 amino acids and spanning the entire protein of interest for the viral- and tumor- associated antigens. For EBV we used EBNA2, EBNA3A/B/C, LMP1/2, and BZLF1 pepmixes; for CMV, pp65 and E1A pepmixes; for adenovirus, penton and hexon pepmixes; for influenza A, H1N1 pepmix (0.6 nmol of each peptide, all from JPT Technologies). We included both negative (DMSO vehicle) and positive (staphylococcal enterotoxin B or PMA/ ionomycin) controls in each experiment. After 16-20 hours of incubation at $37^{\circ} \mathrm{C}$ in $5 \% \mathrm{CO}_{2}$, the plates were developed for IFN- $\gamma^{+}$spots (29), dried overnight at room temperature in the dark, and sent to ZellNet Consulting for quantification. We expressed the frequency of $\mathrm{T}$ cells specific to each antigen as specific spot-forming cells per input cell number.

Statistics. We used descriptive statistics (means, median, ranges, and standard deviations or standard errors) to summarize the data. We used Student's $t$ test (2-tailed) or the nonparametric Wilcoxon rank-sum test for between-group comparisons, while the nonparametric Wilcoxon signed-rank test was applied when observations were made within subject. Pearson correlation was used to test the statistical relationship between 2 variables. A $P$ value less than 0.05 was considered statistically significant.

Study approval. This study was approved by the US FDA, the Recombinant DNA Advisory Committee, and the IRB of Baylor College of Medicine and was conducted in accordance with Declaration of Helsinki principles. All participants provided written informed consent upon enrollment.

\section{Author contributions}

CAR, GD, BG, CMR, MKB, HEH, and BS conceptualized the overall strategy and developed its clinical translation and implementation. The clinical protocol was written by CAR and MKB. CAR was the principal investigator of the protocol, and $\mathrm{BG}, \mathrm{MKB}$, and $\mathrm{HEH}$ were the investigational new drug (IND) sponsors. Manufacturing of $\mathrm{T}$ cells, flow cytometry, and qPCR acquisition of clinical samples were performed by $\mathrm{BB}, \mathrm{HZ}$, and $\mathrm{OD}$, supervised by $\mathrm{BS}$ and $\mathrm{CMR}$, and directed by APG and ZM. The manuscript was written by CAR, BS, and GD, and all authors discussed and interpreted the results. MFW and HL performed statistical analyses. CAR, CMB, BHC, and $\mathrm{HEH}$ enrolled patients in the protocol and/or managed the patients, and MB was the study research nurse, assisting with enrollment, sample acquisition, and data safety monitoring of patients.

\section{Acknowledgments}

We thank the following for financial support: the NIH National Cancer Institute (grants 3P50CA126752 and R01CA131027), the National Heart, Lung, and Blood Institute (R01HL114564), and the Leukemia and Lymphoma Society (LLSTR 6227-08). The clinical trial also received support from the Institute for Clinical and Translational Research at Baylor College of Medicine and shared resources of the Dan L. Duncan Cancer Center support grant 5P30CA125123 (NCI), and the National Gene Vector Biorepository. We also thank Andreas A. Hombach and Hinrich Abken (University of Cologne, Cologne, Germany) for providing the original scFv.

Address correspondence to: Barbara Savoldo, Department of Pediatrics, Lineberger Comprehensive Cancer Center, University of North Carolina, 125 Mason Farm Road 5203, Chapel Hill, North Carolina 27599, USA. Phone: 919.962.8414; E-mail: bsavoldo@med.unc.edu. Or to: Carlos A. Ramos, Center for Cell and Gene Therapy, Baylor College of Medicine, 1102 Bates Street, Suite 1760, Houston, Texas 77030, USA. Phone: 832.824.4817; E-mail: caramos@bcm.edu. 
1. Dotti G, Gottschalk S, Savoldo B, Brenner MK. Design and development of therapies using chimeric antigen receptor-expressing T cells. Immunol Rev. 2014;257(1):107-126.

2. Maude SL, Teachey DT, Porter DL, Grupp SA. CD19-targeted chimeric antigen receptor T-cell therapy for acute lymphoblastic leukemia. Blood. 2015;125(26):4017-4023.

3. Kochenderfer JN, et al. Chemotherapy-refractory diffuse large B-cell lymphoma and indolent B-cell malignancies can be effectively treated with autologous T cells expressing an anti-CD19 chimeric antigen receptor. JClin Oncol. 2015;33(6):540-549.

4. Lee DW, et al. T cells expressing CD19 chimeric antigen receptors for acute lymphoblastic leukaemia in children and young adults: a phase 1 dose-escalation trial. Lancet. 2015;385(9967):517-528.

5. Brentjens RJ, et al. CD19-targeted T cells rapidly induce molecular remissions in adults with chemotherapy-refractory acute lymphoblastic leukemia. Sci Transl Med. 2013;5(177):177ra38.

6. Turtle CJ, et al. Immunotherapy of non-Hodgkin's lymphoma with a defined ratio of $\mathrm{CD} 8^{+}$and $\mathrm{CD} 4^{+}$ CD19-specific chimeric antigen receptor-modified T cells. Sci Transl Med. 2016;8(355):355ra116.

7. Savoldo B, et al. CD28 costimulation improves expansion and persistence of chimeric antigen receptor-modified $\mathrm{T}$ cells in lymphoma patients. JClin Invest. 2011;121(5):1822-1826.

8. Ramos CA, et al. Clinical responses with $\mathrm{T}$ lymphocytes targeting malignancy-associated $\kappa$ light chains. J Clin Invest. 2016;126(7):2588-2596.

9. Porter DL, et al. Chimeric antigen receptor T cells persist and induce sustained remissions in relapsed refractory chronic lymphocytic leukemia. Sci Transl Med. 2015;7(303):303ra139.

10. Wang X, et al. Phase 1 studies of central memory-derived CD19 CAR T-cell therapy following autologous HSCT in patients with B-cell NHL. Blood. 2016;127(24):2980-2990.

11. Maude SL, Barrett D, Teachey DT, Grupp SA. Managing cytokine release syndrome associated with novel $\mathrm{T}$ cell-engaging therapies. Cancer J. 2014;20(2):119-122.

12. Lee DW, et al. Current concepts in the diagnosis and management of cytokine release syndrome. Blood. 2014;124(2):188-195.

13. Davila ML, et al. Efficacy and toxicity management of 19-28z CAR T cell therapy in B cell acute lymphoblastic leukemia. Sci Transl Med. 2014;6(224):224ra25.

14. Falini B, et al. CD30 (Ki-1) molecule: a new cytokine receptor of the tumor necrosis factor receptor superfamily as a tool for diagnosis and immunotherapy. Blood. 1995;85(1):1-14.

15. Younes A, Ansell SM. Novel agents in the treatment of Hodgkin lymphoma: Biological basis and clinical results. Semin Hematol. 2016;53(3):186-189.
16. Forero-Torres A, et al. A phase II study of SGN30 (anti-CD30 mAb) in Hodgkin lymphoma or systemic anaplastic large cell lymphoma. Br J Haematol. 2009;146(2):171-179.

17. Younes A, et al. Brentuximab vedotin (SGN-35) for relapsed CD30-positive lymphomas. N Engl J Med. 2010;363(19):1812-1821.

18. Moskowitz $\mathrm{CH}$, et al. Brentuximab vedotin as consolidation therapy after autologous stemcell transplantation in patients with Hodgkin's lymphoma at risk of relapse or progression (AETHERA): a randomised, double-blind, placebo-controlled, phase 3 trial. Lancet. 2015;385(9980):1853-1862.

19. Bollard CM, et al. Cytotoxic T lymphocyte therapy for Epstein-Barr virus+ Hodgkin's disease. J Exp Med. 2004;200(12):1623-1633.

20. Bollard CM, et al. Sustained complete responses in patients with lymphoma receiving autologous cytotoxic T lymphocytes targeting Epstein-Barr virus latent membrane proteins. JClin Oncol. 2014;32(8):798-808.

21. Roskrow MA, et al. Epstein-Barr virus (EBV)specific cytotoxic $\mathrm{T}$ lymphocytes for the treatment of patients with EBV-positive relapsed Hodgkin's disease. Blood. 1998;91(8):2925-2934.

22. Younes A, et al. Nivolumab for classical Hodgkin's lymphoma after failure of both autologous stem-cell transplantation and brentuximab vedotin: a multicentre, multicohort, single-arm phase 2 trial. Lancet Oncol. 2016;17(9):1283-1294.

23. Ansell SM, et al. PD-1 blockade with nivolumab in relapsed or refractory Hodgkin's lymphoma. N Engl J Med. 2015;372(4):311-319.

24. Armand P, et al. Programmed death-1 blockade with pembrolizumab in patients with classical hodgkin lymphoma after brentuximab vedotin failure. J Clin Oncol. 2016;34(31):3733-3739.

25. Wang CM, et al. Autologous T cells expressing $\mathrm{CD} 30$ chimeric antigen receptors for relapsed or refractory Hodgkin lymphoma: an open-label phase I trial. Clin Cancer Res. 2017;23(5):1156-1166.

26. Savoldo B, et al. Epstein Barr virus specific cytotoxic $\mathrm{T}$ lymphocytes expressing the anti$\mathrm{CD} 30 \zeta$ artificial chimeric T-cell receptor for immunotherapy of Hodgkin disease. Blood. 2007;110(7):2620-2630.

27. Di Stasi A, et al. T lymphocytes coexpressing CCR4 and a chimeric antigen receptor targeting CD30 have improved homing and antitumor activity in a Hodgkin tumor model. Blood. 2009;113(25):6392-6402.

28. Croft M. Co-stimulatory members of the TNFR family: keys to effective T-cell immunity? Nat Rev Immunol. 2003;3(8):609-620.

29. Arber C, et al. The immunogenicity of virusderived $2 \mathrm{~A}$ sequences in immunocompetent indi- viduals. Gene Ther. 2013;20(9):958-962.

30. Zanotti R, et al. Serum levels of soluble CD30 improve International Prognostic Score in predicting the outcome of advanced Hodgkin's lymphoma. Ann Oncol. 2002;13(12):1908-1914.

31. Hombach A, et al. An anti-CD30 chimeric receptor that mediates CD3-zeta-independent T-cell activation against Hodgkin's lymphoma cells in the presence of soluble CD30. Cancer Res. 1998;58(6):1116-1119.

32. Aldinucci D, Gloghini A, Pinto A, De Filippi R, Carbone A. The classical Hodgkin's lymphoma microenvironment and its role in promoting tumour growth and immune escape. J Pathol. 2010;221(3):248-263.

33. Turtle CJ, et al. CD19 CAR-T cells of defined $\mathrm{CD} 4^{+}: \mathrm{CD} 8^{+}$composition in adult $\mathrm{B}$ cell ALL patients. JClin Invest. 2016;126(6):2123-2138.

34. Maude SL, et al. Chimeric antigen receptor $\mathrm{T}$ cells for sustained remissions in leukemia. $N$ Engl JMed.2014;371(16):1507-1517.

35. Kochenderfer JN, et al. B-cell depletion and remissions of malignancy along with cytokineassociated toxicity in a clinical trial of anti-CD19 chimeric-antigen-receptor-transduced $\mathrm{T}$ cells Blood.2012;119(12):2709-2720.

36. Klebanoff CA, Khong HT, Antony PA, Palmer DC, Restifo NP. Sinks, suppressors and antigen presenters: how lymphodepletion enhances $\mathrm{T}$ cell-mediated tumor immunotherapy. Trends Immunol. 2005;26(2):111-117.

37. Slivnick DJ, Ellis TM, Nawrocki JF, Fisher RI. The impact of Hodgkin's disease on the immune system. Semin Oncol. 1990;17(6):673-682.

38. Bollard CM, et al. Complete responses of relapsed lymphoma following genetic modification of tumor-antigen presenting cells and T-lymphocyte transfer. Blood. 2007;110(8):2838-2845.

39. Jensen MC, et al. Antitransgene rejection responses contribute to attenuated persistence of adoptively transferred CD20/CD19specific chimeric antigen receptor redirected $\mathrm{T}$ cells in humans. Biol Blood Marrow Transplant. 2010;16(9):1245-1256.

40. Hudecek M, et al. The nonsignaling extracellular spacer domain of chimeric antigen receptors is decisive for in vivo antitumor activity. Cancer Immunol Res. 2015;3(2):125-135.

41. Reichel J, et al. Flow sorting and exome sequencing reveal the oncogenome of primary Hodgkin and Reed-Sternberg cells. Blood. 2015;125(7):1061-1072.

42. Cheson BD, et al. Revised response criteria for malignant lymphoma. JClin Oncol. 2007;25(5):579-586.

43. Xu Y, et al. Closely related T-memory stem cells correlate with in vivo expansion of CAR.CD19-T cells and are preserved by IL-7 and IL-15. Blood. 2014;123(24):3750-3759. 